\title{
Coincident Bilateral Atraumatic Hip Fracture in a Young Patient with Renal Osteodystrophy
}

\author{
Shukriah Aqilah Zakaria ${ }^{*}$, Mohd Shafiee Johan Chin ${ }^{2}$, Max Yong Guang Yi ${ }^{1}$, \\ Mohd Atiq Che Roselam1, Mohamad Hafiz Mohmad Hassim³ \\ ${ }^{1}$ Department of Orthopaedic, Hospital Bintulu, Bintulu, Malaysia \\ ${ }^{2}$ Department of Orthopaedic, Hospital Kuala Lumpur, Kuala Lumpur, Malaysia \\ ${ }^{3}$ Department of Orthopaedic, Hospital Tuanku Jaafar, Seremban, Malaysia \\ Email: *shuqzakaria@gmail.com
}

How to cite this paper: Zakaria, S.A. Chin, M.S.J., Yi, M.Y.G., Roselam, M.A.C. and Hassim, M.H.M. (2020) Coincident Bilateral Atraumatic Hip Fracture in a Young Patient with Renal Osteodystrophy. Open Journal of Orthopedics, 10, 313-320. https://doi.org/10.4236/ojo.2020.1011032

Received: October 14, 2020

Accepted: November 23, 2020

Published: November 26, 2020

Copyright (C) 2020 by author(s) and Scientific Research Publishing Inc. This work is licensed under the Creative Commons Attribution International License (CC BY 4.0).

http://creativecommons.org/licenses/by/4.0/

\begin{abstract}
Bilateral, coincident, atraumatic hip fracture is extremely uncommon and usually occurs secondary to seizures, trauma and metabolic disease including renal osteodystrophy. One of the major types, secondary hyperparathyroidism is associated with high bone turnover due to excess parathyroid hormone and usually seen in a chronic dialysis patient. We reported a 20 -year-old woman with end stage renal failure and renal osteodystrophy. She sustained atraumatic right subtrochanteric fracture and left neck of femur fracture (Garden 1), then underwent bilateral long proximal femoral nail. Renal osteodystrophy causes pathological fracture by affecting calcium metabolism that stimulates bone resorptions and leads to osteoporotic bone. The aim of this case report is to discuss the approach and management done to the patient presented to our center. Bilateral long proximal femoral nail (PFN) was chosen, taking into account the patient's premorbid, age, fracture pattern and potential complications. Careful multidisciplinary team approach led by the orthopaedic surgeon, nephrologist and physical therapist is vital for the patient to achieve good outcome postoperatively, thus reducing morbidity and mortality.
\end{abstract}

\section{Keywords}

Pathological Fracture, Hip Fracture, Secondary Hyperparathyroidism, Renal Osteodystrophy

\section{Introduction}

Bilateral hip fracture is extremely uncommon, even more infrequent to occur at such a young age and without trauma history. It usually affects those with sei- 
zures, trauma and metabolic disease including renal osteodystrophy, which is associated with many skeletal complications including osteonecrosis, osteochondritis dissecans, osteomalacia, osteosclerosis and developmental disorders in children [1]. One of the major types, secondary hyperparathyroidism is associated with marked parathyroid hyperplasia causing excess production of parathyroid hormone (PTH). This is the major feature seen in a chronic dialysis patient [2]. Pathological fracture is common in this group. According to Coco and Rush [3], hemodialysis patients have 17.4 and 2.4 times higher incidence rates of hip fracture than non-hemodialysis patients. However, simultaneous hip fractures are very rare [4]. In this case report, we will be discussing how renal osteodystrophy can cause pathological fracture and the management done for this patient.

\section{Case Report}

A 20-year-old woman was admitted to the medical ward for infective acute gastroenteritis with underlying end-stage renal failure secondary to rapid progressive glomerulonephritis. The patient has been on hemodialysis for the past 7 years and had a history of admission to the orthopedic ward for traumatic L5 pars interarticularis fracture and bilateral superior pubic rami and right inferior pubic rami fracture. Both were treated conservatively. She was referred to orthopedic team during her stay for sudden onset of bilateral hip pain upon walking up to the ambulance after a dialysis session with absent history of trauma. Examination revealed externally rotated right hip with tenderness over the right greater trochanter and tenderness over her left proximal thigh. Range of movement (ROM) bilateral hip restricted due to pain. Neurovascular examinations of bilateral lower limbs were unremarkable. Pelvic radiograph (Figure 1) showed right subtrochanteric fracture and left neck of femur fracture (Garden 1). Her bilateral femurs were generally osteoporotic with plain radiography showed thinned out cortex and loss of trabecular lines over the midshaft and distal femur.

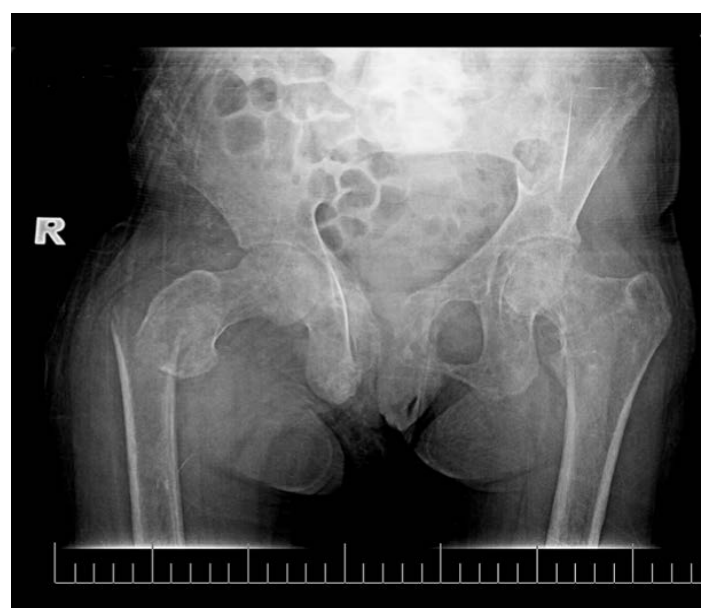

Figure 1. Plain radiograph of bilateral hip and pelvis in anteroposterior (AP) showing right subtrochanteric fracture and Garden 1 left neck of femur fracture. 
She was also diagnosed to have secondary hyperparathyroidism with a definitive plan for parathyroidectomy later. Blood investigations showed normal calcium level with elevated phosphate, alkaline phosphatase (ALP) and parathyroid hormone (PTH). Osteosynthesis with bilateral long proximal femoral nail was done in two staged operations and stability achieved (Figures 2-4). The patient was subsequently discharged home well few days postoperatively. Calcium supplements and vitamin $\mathrm{D}$ treatments were given for the prevention of future comorbidity. She was also referred to the physiotherapy department for muscle strengthening and range of motion exercise. She was kept without bearing weight on her both lower limbs and was evaluated routinely initially one monthly under outpatient clinic. The tenth month post operation, union achieved radiographically and clinically (Figures 5-7) with full ROM of bilateral hips. The patient was able to ambulate with full weight bearing supported with crutches with her next follow up scheduled 2 years post osteosynthesis to review her conditions.

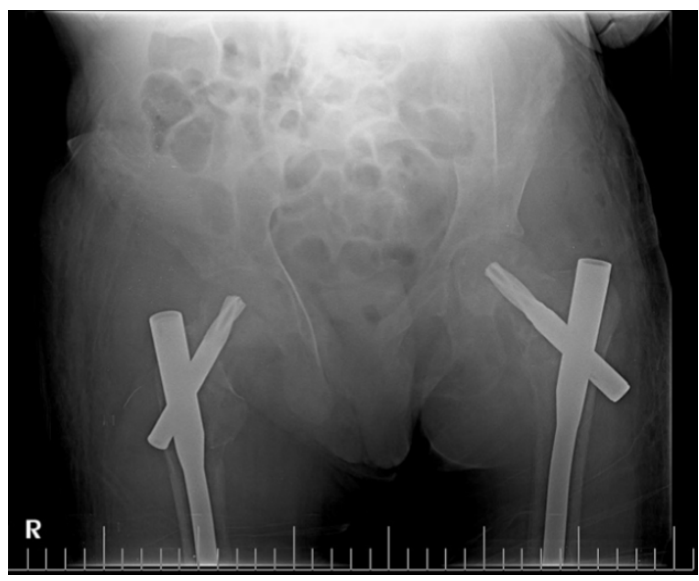

Figure 2. Pelvic X-ray anteroposterior (AP) view post bilateral long proximal femoral nail (PFN) insertion.

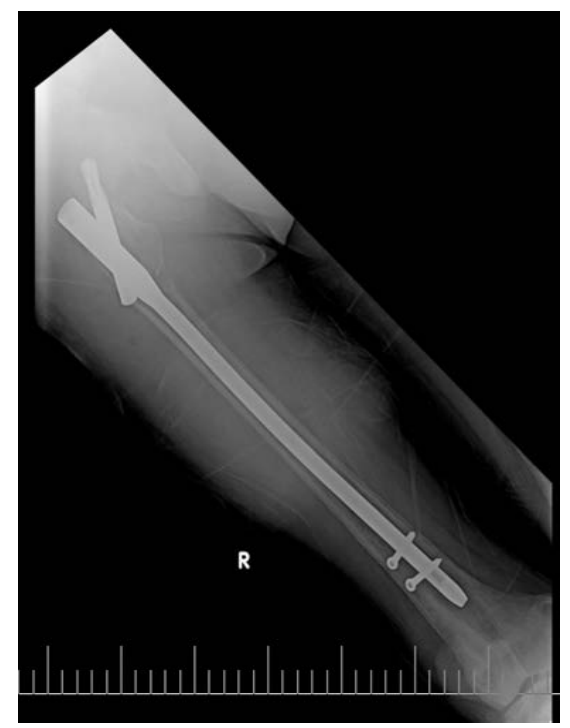

Figure 3. Right femur lateral view with long PFN. 


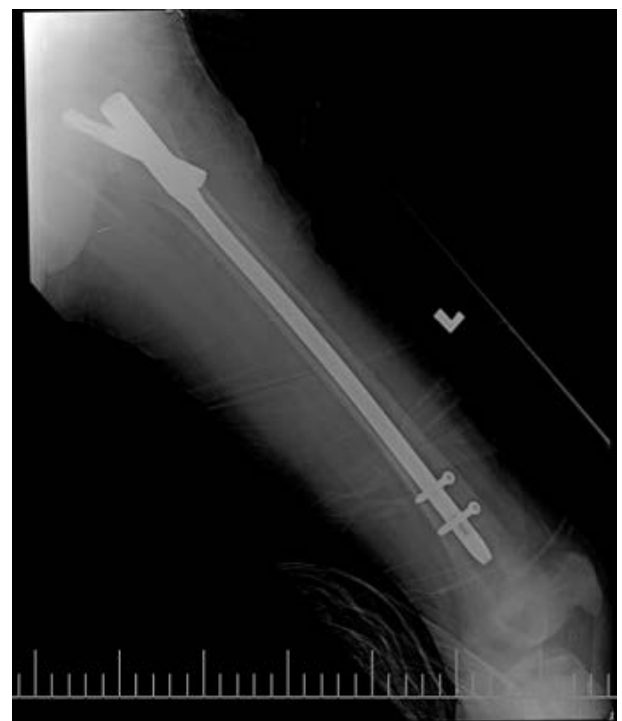

Figure 4. Left femur lateral view with long PFN.

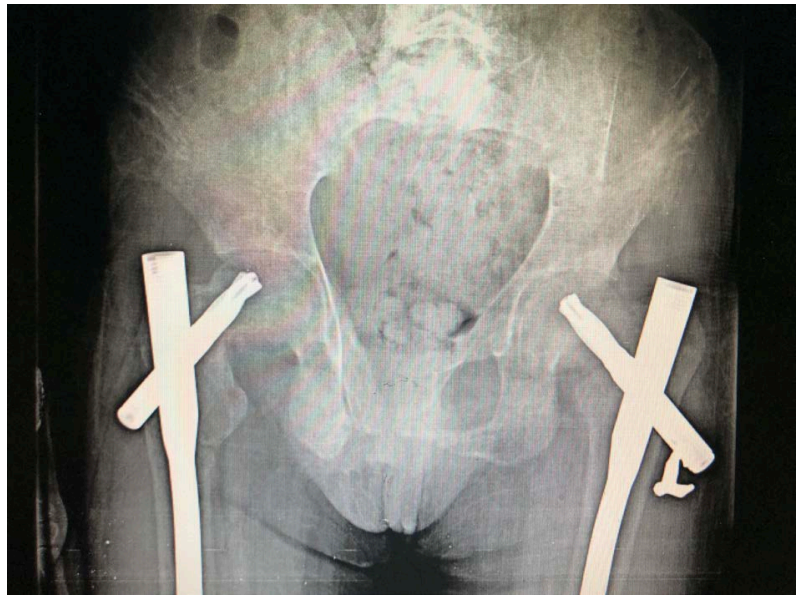

Figure 5. Pelvic X-ray anteroposterior (AP) view, 10 months post bilateral long proximal femoral nail (PFN) insertion.

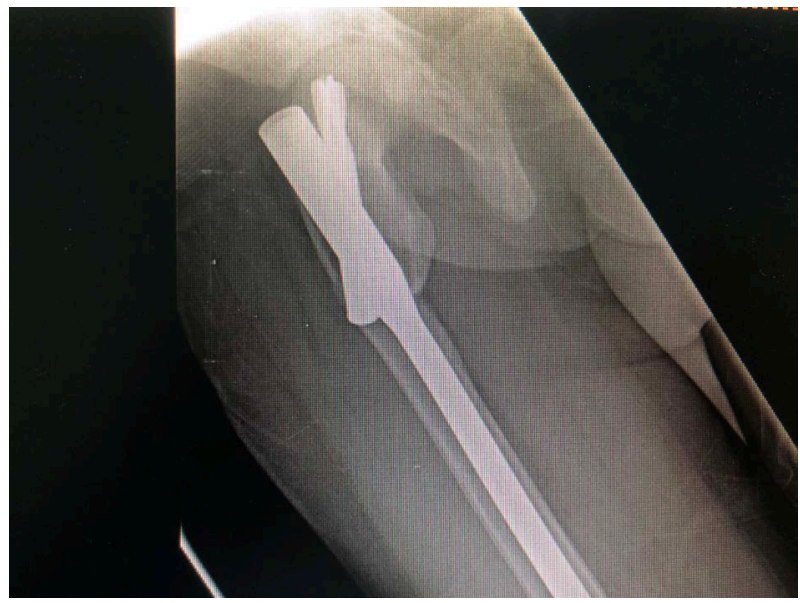

Figure 6. Right hip X-ray lateral view, 10 months post bilateral long proximal femoral nail (PFN) insertion. 


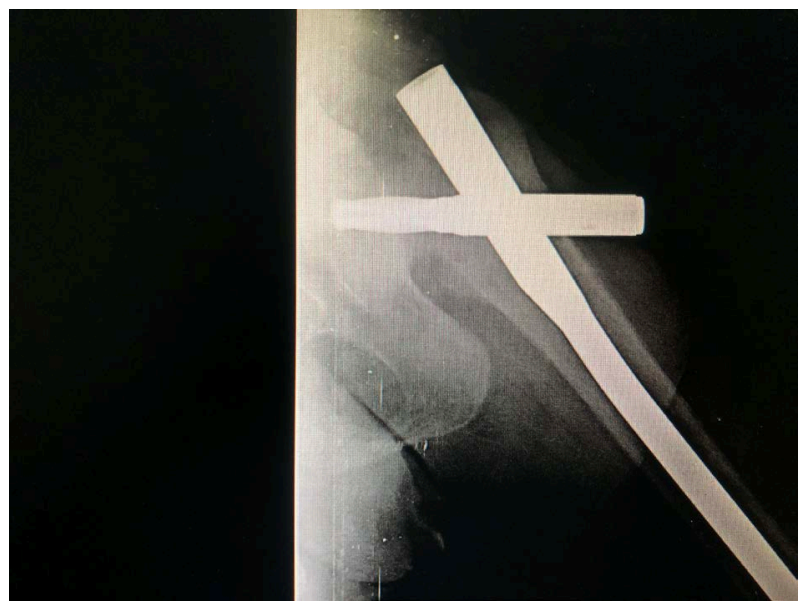

Figure 7. Left hip X-ray lateral view, 10 months post bilateral long proximal femoral nail (PFN) insertion.

\section{Discussion}

In a healthy adult bone, bilateral hip fractures are relatively rare, as it requires significant trauma such as fall from height and motor vehicle accident. The hip is an area where the torque of powerful countering muscles is nicely balanced. The femoral neck serves as the lever arm with the center of the femoral head act as the center of rotation of the femoral joint that carries all the acting muscle and weight bearing forces. Anything that caused deterioration of the bone structure or quality makes this area prone to fractures [5].

Based on the anatomic location, hip fractures can be classified into two major types which are femoral neck fractures and trochanteric fractures [6]. The femoral neck fracture is classified by Garden according to displacement and the type 1 is incomplete, undisplaced fracture. Meanwhile, trochanteric fracture involves between the cervical region of proximal femur and the shaft. A fracture line running from an area within $5 \mathrm{~cm}$ distal to the lesser trochanter is known as subtrochanteric fracture.

In cases of pathological fracture, it is usually associated with neoplastic disease, metabolic disease and renal osteodystrophy [7]. Pathophysiology on how renal osteodystrophy causes osteoporotic bone begins with the failure of filtration and hence results in phosphate retentions. Hyperphosphataemia causes a decrease in vitamin 1,25-dihydroxyvitamin $\mathrm{D}$ synthesis which then leads to decrease absorption of calcium from the intestinal and kidney. Low serum calcium level will stimulate the parathyroid gland to secrete more parathyroid hormone (PTH) and eventually will stimulate osteoclastic action and bone resorptions that subsequently will lead to osteoporotic bone [8].

In renal failure patient with hemodialysis sustaining hip fracture, recent reports favor osteosynthesis, and it was found to be superior to conservative management due to higher mortality in those patients managed conservatively [9]. Although some advocate for conservative management for impacted undisplaced femoral neck fracture, Bently [10] found that this fracture will displace as 
much as $15 \%$ most of the time. As stated by Klein et al, operative treatment and aggressive mobilization allow patients with renal failure to return to premorbid ambulation [11].

For hip fracture in pathologic bone, internal fixation is the most satisfactory treatment in either displaced or undisplaced fractures with good bone stock. The preferred method includes cannulated screw and dynamic hip screw (DHS) or in the older patient with underlying hip disease, head sacrificing surgery such as cemented hemiarthroplasty or total hip arthroplasty are strongly recommended. However, in the young patient, pre-injury status and life expectancy are the key factors when determining whether to proceed with head sacrificing or head salvaging surgery. Albeit prosthetic replacement allows patient's early return to ambulation post operatively, it carries the risks of loosening of the prosthesis and subsequent revision surgery [5]. In a meta-analysis of 106 reports pertaining to the femoral neck fracture, Lu-Yao et al. [12] showed that although internal fixation is associated with a higher failure rate, it offers the long-term chance of regaining normal function of the hip.

In this patient, a cephalomedullary device, long proximal femoral nail (PFN) was chosen rather than short PFN as prophylactic fixation mainly due to patient age factor and osteoporotic bone condition. Patient with renal osteodystrophy have abnormal biomechanical parameters and it is much fragile and brittle, making it more difficult to achieve a stable implant-bone construct needed for fracture fixation. Relative stability technique reduces the risk of failure at the bone-implant interface and this can be achieved by load sharing implants which is PFN [5]. Although there is no established guideline on choosing long or short nails, but a literature mentioned that long nails would avoid diaphyseal stress risers and reduce the rate of periprosthetic fractures [13] [14] [15]. In the case of primary bone disease at her age with normal life expectancy, the use of a hip prosthesis is not preferred because we have to choose the least invasive procedure in order to retain maximum bone stock, bear in mind the possibility of revision surgery in the future [5].

A treatment regimen should be planned, aiming to regain at least near-normal bone mass in order to help with the bone healing and to prevent subsequent osteoporotic fractures and complications associated with operation. This is executed by oral supplements such as calcium, vitamin D analogues and bisphosphonates, other than regular dialysis. Parathyroidectomy was planned for this patient as a definitive treatment to cure the underlying disease. Apart from the medical treatment, comprehensive intervention including physiotherapy, occupational therapy, and fall prevention may also prevent secondary fracture after fixation [16].

\section{Conclusion}

Atraumatic bilateral hip fracture is rare but increasingly being reported in the patient with chronic renal disease. It is associated with high mortality and mor- 
bidity. High index of suspicion is needed to detect any fracture as the patient is mostly bed bounded and it can be easily missed out. These fractures can be prevented by early diagnosis of chronic renal disease and strict optimization of metabolic parameters to avoid progression to severe osteoporosis. Understanding the potential complications according to the fracture type and the treatment modality used is important, so that the correct primary fixation is chosen. In the young patient, preservation of femoral head is the most important. Management should be tailored according to the patient's premorbid condition and severity of metabolic derangements with careful multidisciplinary team approach in order to prevent further complications.

\section{Conflicts of Interest}

The authors declare no conflicts of interest regarding the publication of this paper.

\section{References}

[1] Crutchlow, W.P. and David, D. S. (1971) Skeletal Complications of Kidney Disease. Clinical Orthopaedics and Related Research, 74, 209-220. https://doi.org/10.1097/00003086-197101000-00028

[2] Kurokawa, K. and Fukagawa, M. (1999) Uremic Bone Diseases: Advances over the Last 30 Years. Journal of Nephrology, 12, S63-S67.

[3] Coco, M. and Rush, H. (2000) Increased Incidence of Hip Fractures in Dialysis Patients with Low Serum Parathyroid Hormone. American Journal of Kidney Diseases, 36, 1115-1121. https://doi.org/10.1053/ajkd.2000.19812

[4] Ogun, T.C., Memik, R., Yel, M. and Sarlak, A. (2001) Bilateral Pathologic Femoral Neck Fracture as a Consequence of Ranalosteodystrophy: Report of Two Cases and Review of the Literature. Journal of Arthroplasty \& Arthroscopy Surgery, 12, 199-202.

[5] Mataliotakis, G., Lykissas, M.G., Mavrodontidis, A.N., Kontogeorgakos, V.A. and Beris, A.E. (2009) Femoral Neck Fractures Secondary to Renal Osteodystrophy. Literature Review and Treatment Algorithm. Journal of Musculoskeletal and Neuronal Interactions, 9, 130-137.

[6] Hung, L.W., Hwang, Y.T., Huang, G.S., Liang, C.C. and Lin, J. (2017) The Influence of Renal Dialysis and Hip Fracture Sites on the 10-Year Mortality of Elderly Hip Fracture Patients: A Nationwide Population-Based Observational Study. Medicine, 96, e7618. https://doi.org/10.1097/MD.0000000000007618

[7] Negishi, H., Kobayashi, M., Nishida, R., Yamada, H., Ariga, S., Sasaki, F. and Fujimoto, S. (2002) Primary Hyperparathyroidism and Simultaneous Bilateral Fracture of the Femoral Neck during Pregnancy. The Journal of Trauma: Injury, Infection, and Critical Care, 52, 367-369. https://doi.org/10.1097/00005373-200202000-00026

[8] Mankin, H.J. (1974) Rickets, Osteomalacia and Renal Osteodystrophy (Review Article, Part 1). Journal of Bone \& Joint Surgery, 56, 101-128.

https://doi.org/10.2106/00004623-197456010-00012

[9] Schaab, P.C., Murphy, G., Tzamaloukos, A.H., Hays, M.B., Merlin, T.L., Eisenberg, B., Avasthi, P.S. and Worrell, R.V. (1990) Femoral Neck Fractures in Patients Receiving Long-Term Dialysis. Clinical Orthopaedics and Related Research, 260, 224-231. https://doi.org/10.1097/00003086-199011000-00038 
[10] Bentley, G. (1980) Treatment of Nondisplaced Fractures of the Femoral Neck. Clinical Orthopaedics and Related Research, 152, 93-101. https://doi.org/10.1097/00003086-198010000-00010

[11] Klein, D.M., Tornetta, P., Barbera, C. and Neuman, D. (1998) Operative Treatment of Hip Fractures in Patients with Renal Failure. Clinical Orthopaedics and Related Research, 350, 174-178. https://doi.org/10.1097/00003086-199805000-00024

[12] Lu-Yao, G.L., Keller, R.B., Littenberg, B. and Wennberg, J.E. (1994) Outcomes after Displaced Fractures of the Femoral Neck: A Meta-Analysis of One Hundred and Six Published Reports. Journal of Joint \& Bone Surgery, 76, 15-25. https://doi.org/10.2106/00004623-199401000-00003

[13] Knobe, M., Münker, R., Sellei, R.M., Schmidt-Rohlfing, B., Erli, H.J., Strobl, C.S. and Niethard, F.U. (2009) Unstable Periintertrochanteric Femur Fractures. Failure Rate, Lag Screw Sliding and Outcome with Extra and Intramedullary Devices (PCCP, DHS and PFN). Zeitschrift für Orthopädie und Unfallchirurgie, 147, 306-313. https://doi.org/10.1055/s-0029-1185349

[14] Wirtz. C., Abbassi, F., Evangelopoulos, D.S., Kohl, S., Siebenrock, K.A. and Krüger, A. (2013) High Failure Rate of Intertrochanteric Fracture Osteosynthesis with Proximal Femoral Locking Compression Plate. Injury, 44, 751-756. https://doi.org/10.1016/j.injury.2013.02.020

[15] Shen, L., Zhang, Y., Shen, Y. and Cui, Z. (2013) Antirotation Proximal Femoral Nail versus Dynamic Hip Screw for Interintertrochanteric Fractures: A Meta-Analysis Ofrandomized Controlled Studies. Orthopaedics \& Traumatology: Surgery \& Research, 99, 377-383. https://doi.org/10.1016/j.otsr.2012.12.019

[16] Yoon, B.H. and Koo, K.H. (2017) Hip Fracture in Chronic Kidney Disease Patients: Necessity of Multidisciplinary Approach. Journal of Korean Medical Science, 32, 1906-1907. https://doi.org/10.3346/jkms.2017.32.12.1906 